\title{
Using Accelerometer/GPS Data to Validate a Neighborhood-Adapted Version of the International Physical Activity Questionnaire (IPAQ)
}

\author{
Levi Frehlich, Christine Friedenreich, and Alberto Nettel-Aguirre \\ University of Calgary \\ Jasper Schipperijn \\ University of Southern Denmark \\ Gavin R. McCormack \\ University of Calgary
}

\begin{abstract}
Despite continued interest in neighborhood correlates of physical activity, few self-report questionnaires exist that capture neighborhood-based physical activity. Furthermore, there is little evidence about the measurement validity of self-report measures of neighborhood-based physical activity. Notably, self-reported neighborhood physical activity has not been validated against combined accelerometer and global positioning system (GPS)-assessed physical activity. Thus, the purpose of this study was to estimate the concurrent validity of a recently adapted tool for capturing self-reported neighborhood-based physical activity (i.e., the Neighborhood International Physical Activity Questionnaire; N-IPAQ). Adults $(n=75)$ from four Calgary (Alberta, Canada) neighborhoods wore an accelerometer and GPS monitor for 7 consecutive days after which they self-reported their physical activity from the past week using the N-IPAQ. Bland-Altman plots and Spearman correlations estimated the concurrent validity between N-IPAQ and accelerometer/GPS physical activity (estimated for the administrative boundary, 400-m and 800-m radial buffers). The mean (95\% Confidence Interval [CI]) difference between the N-IPAQ and accelerometer/GPS estimated total daily minutes of physical activity differed for the $400-\mathrm{m} \mathrm{(1.9} \mathrm{min},-26.2$ to 29.9$), 800-\mathrm{m}$ (10.6 min, -16.0 to 37.1$)$, and administrative boundary buffers (14.7 $\mathrm{min},-11.5$ to 41.0$)$. The strongest Spearman correlations were found between the N-IPAQ and 800-m radial buffer accelerometer-captured vigorous-intensity physical activity ( $r=.41$ [95\% CI: .18 to .60]), and the N-IPAQ and administrative boundary accelerometer-captured vigorous-intensity physical activity ( $r=.43$ [95\% CI: .20 to .62]). Our findings suggest that the N-IPAQ provides good estimates of neighborhood-based physical activity and could be used when investigating neighborhood correlates of physical activity.
\end{abstract}

Keywords: built environment, community, GIS, monitoring, self-report, survey

Evidence regarding the role of neighborhood built characteristics in supporting physical activity continues to accumulate (Barnett et al., 2017; Wasfi, Steinmetz-Wood, \& Kestens, 2017) and inform urban planning and policy (Calise, Heeren, DeJong, Dumith, \& Kohl, 2013; Kärmeniemi, Lankila, Ikäheimo, Koivumaa-

(C) 2018 The Authors. Published by Human Kinetics, Inc. This is an Open Access article distributed under the terms of the Creative Commons Attribution License CC BY NC 4.0, which permits unrestricted noncommercial use, distribution, and reproduction in any medium, provided the original work is properly cited, the new use includes a link to the license, and any changes are indicated. See http:// creativecommons.org/licenses/by-nc/4.0. This license does not cover any third-party material which may appear with permission in the article. For commercial use, permission should be requested from Human Kinetics, Inc., through the Copyright Clearance Center (http://www.copyright.com).

Frehlich, Friedenreich, Nettel-Aguirre, and McCormack are with the Dept. of Community Health Sciences, Cumming School of Medicine, University of Calgary, Calgary, Alberta, Canada. Schipperijn is with the Dept. of Sport Sciences and Clinical Biomechanics, University of Southern Denmark, Odense, Denmark. Address author correspondence to Levi Frehlich at lcfrehli@ucalgary.ca.
Honkanen, \& Korpelainen, 2018). In adults, consistent associations between land-use mix, population density, street connectivity, and physical activity have been found (Cerin et al., 2017; Kärmeniemi et al., 2018; Sallis et al., 2016). However, most studies that have contributed to this evidence have relied on self-report measures that do not take into account the location in which physical activity is undertaken (Ferdinand, Sen, Rahurkar, Engler, \& Menachemi, 2012). Few neighborhood-based self-report physical activity measures exist, and despite demonstrating adequate reliability (Bae, Cho, \& Son, 2015; Giles-Corti et al., 2006; McCormack et al., 2009) none, to our knowledge, have been evaluated for concurrent validity. Thus, the extent to which neighborhood characteristics are associated specifically with neighborhood physical activity is not well quantified. More development and testing of self-report questionnaires that accurately capture physical activity within locations of interest (i.e., neighborhoods) are necessary for better local urban planning policies and public health interventions (Jansen, Kamphuis, Pierik, Ettema, \& Dijst, 2018).

Existing self-report neighborhood-based physical activity tools have typically measured "usual" weekly physical activity to capture 
habitual behavior (Giles-Corti et al., 2006). Usual weekly neighborhood physical activity is found to be higher in neighborhoods with increased land-use mix, population density, street connectivity (Christian et al., 2011), and higher walkability (McCormack et al., 2012). Furthermore, changes in usual neighborhood physical activity have been detected after residential relocation (Calise et al., 2013; Giles-Corti et al., 2013). Nevertheless, measures of usual physical activity may be less sensitive to the immediate effects of neighborhood interventions - at least in the short-term, because respondents may report their average or usual behavior for many weeks or even months, rather than reporting the physical activity they undertook in the weeks immediately before and after an intervention (Doma, Speyer, Leicht, \& Cordier, 2017). Recently, we adapted and tested the measurement properties of the International Physical Activity Questionnaire (IPAQ) to capture neighborhoodbased physical activity undertaken in the last week, finding that the self-administered tool had moderate to excellent test-retest reliability (Frehlich, Friedenreich, Nettel-Aguirre, \& McCormack, 2018). The IPAQ is a non-location specific physical activity tool that has acceptable reliability and moderate concurrent validity when compared with accelerometers (Cerin et al., 2016; Craig et al., 2003). Further, observational (Sundquist et al., 2011) and natural experiment (MacMillan et al., 2018) studies have found associations between IPAQ measured physical activity and neighborhood built characteristics.

Different objective measures (e.g., accelerometers, pedometers, direct observation, and diaries) have been used to assess the concurrent validity of self-report physical activity questionnaires (Doma et al., 2017). However, one challenge of assessing the concurrent validity of neighborhood-based physical activity is that in addition to an objective measure of physical activity, there must also be a measure of geographical location. Recent studies have captured location specific physical activity through the use of Global Position Systems (GPS) monitors usually linked via time stamp with accelerometer data (Jansen et al., 2018; Troped, Wilson, Matthews, Cromley, \& Melly, 2010). GPS linked accelerometer data have been used to assess physical activity in the workplace, commute routes, and home neighborhoods (Krenn, Titze, Oja, Jones, \& Ogilvie, 2011). GPS and accelerometer linked data have also been overlaid with Geographic Information Systems (GIS) to research built environment characteristics (Jansen et al., 2018; Troped et al., 2010). Linking GPS, GIS, and accelerometer data allows for investigations into how different definitions of neighborhoods and their characteristics influence physical activity (Jansen et al., 2018).

The purpose of our study was to evaluate the concurrent validity of a modified self-report neighborhood-based physical activity questionnaire (i.e., the neighborhood-adapted version of the IPAQ [N-IPAQ]) (Frehlich et al., 2018). Specifically, we estimated the correspondence between accelerometer/GPS captured neighborhood-based physical activity and self-reported neighborhoodbased physical activity from the N-IPAQ. Further, we evaluated the extent to which estimates of concordance were sensitive to the size of objectively defined neighborhoods (including administrative boundary, 400-m, and 800-m radial buffers).

\section{Methods}

\section{Study Design and Sample Recruitment}

Our study included a convenience sample $(n=75)$ of adults $(>18$ years of age) drawn from a pool of participants who, in 2014, participated in a Calgary (Canada) study (The Pathways to Health Study; McCormack et al., 2013; 2018). The Pathways to Health study included stratified random sampling to recruit participants $(n=1023)$ from 12 neighborhoods with different street patterns (grid, warped-grid, and curvilinear) and socioeconomic status (quartiles: low, low-medium, high-medium, and high). Socioeconomic status was estimated based on a deprivation index calculated based on a composite score from seven Canadian Census variables. Further details about the Pathways to Health Study methodology is presented elsewhere (McCormack et al., 2018; McInerney et al., 2016).

For the current study, we re-recruited participants who had agreed to be contacted in the future to undertake further research. Specifically, we recruited from those who resided in two lowmedium and two high-medium socioeconomic status (SES) neighborhoods. From these four neighborhoods, we contacted 211 participants via email or telephone, of which 75 agreed to participate and provided written informed consent.

\section{Data Collection}

Data were collected directly from participants in their homes on two occasions approximately 7 days apart. During the first home visit, the participant completed a self-administered N-IPAQ (including sociodemographic items) measuring perceived residential neighborhoodbased physical activity, and was provided with written and verbal instructions along with a practical demonstration on the appropriate wear and use of the GPS and accelerometer monitors. Both monitors were attached to a single elastic belt and participants were instructed to wear the GPS above the left hip and the accelerometer above the right hip. Participants wore the monitors for 7 consecutive days, during all waking hours, except during activities that could potentially damage the monitors (i.e., impact sports or water-based activities). During the second visit, the N-IPAQ was completed again and the GPS and accelerometer monitors were collected. The N-IPAQ was administered twice to assess test-retest reliability; these results have been reported previously (Frehlich et al., 2018). Validity assessment used results from the second administration. The University of Calgary Conjoint Health Research Ethics Board approved this study (Ethics ID: REB15-2940).

\section{Measures}

Sociodemographic Characteristics. Self-reported sociodemographic questions captured sex, age, dependents $<6$ and $6-18$ years old at home, dog ownership, motor vehicle access for personal use (all or some of the time vs. not at all or don't drive), bicycle access for personal use (yes vs. no), and education attainment (university vs. less than university).

The Neighborhood International Physical Activity Questionnaire. We modified the IPAQ (long-form) items to capture self-reported physical activity undertaken inside the neighborhood during the last 7 days (Supplementary Material [available online]). Neighborhood physical activity captured included walking for transportation, bicycling for transportation, walking for leisure, moderate-intensity physical activity, and vigorous-intensity physical activity. Some previous questionnaires capturing neighborhood physical activity provide respondents with an operational definition of neighborhood (e.g., Neighbourhood Physical Activity Questionnaire: 10 to 15-minute walk from home) (Giles-Corti et al., 2006) whereas other have not explicitly defined neighborhood for respondents (Humpel et al., 2004; Sundquist et al., 2011). 
Recognizing that participants may define their neighborhood boundaries differently, we also did not restrict neighborhood to a single operational definition in the N-IPAQ, asking participants to report physical activity in their own perceived neighborhood. Our decision to not be stringent in the geographical definition of the neighborhood was to provide a tool that could be used more generally for neighborhoods of different sizes. We acknowledge, however, that although this approach is useful in terms of practicality, it may have resulted in lower than expected agreement between the N-IPAQ and accelerometer/GPS measured physical activity. Further, it is also possible that some participants worked in their home neighborhoods, which is unlikely to impact agreement between N-IPAQ and accelerometer/GPS measure physical activity, but could mean higher intra-neighborhood physical activity and or fewer inter-neighborhood walking or cycling trips. It should also be noted that if participants work from home or in their perceived neighborhood, they may have been better able to report certain trips over those who do not. For example, if a participant worked outside their perceived neighborhood they may not have reported the portion of the trip to and from work that took place in their neighborhood. Consistent with previous neighborhood physical activity studies using the IPAQ, outlier values for physical activity variables captured using the N-IPAQ were truncated at the 99th percentile of our sample (Cerin, Saelens, Sallis, \& Frank, 2006).

From the N-IPAQ, we used self-reported minutes per day of moderate and vigorous-intensity physical activity and calculated selfreported total daily neighborhood physical activity by summing all self-reported daily physical activity. Daily minutes of neighborhood total walking (walking for leisure plus walking for transportation), moderate-intensity physical activity plus walking (moderate-intensity physical activity plus total walking), moderate-to-vigorous intensity physical activity (moderate- plus vigorous-intensity physical activity), moderate-to-vigorous-intensity physical activity plus walking (moderate-to-vigorous intensity physical activity plus total walking), and total physical activity (moderate-to-vigorous intensity physical activity plus total walking plus bicycling for transportation) were also calculated from the N-IPAQ.

Accelerometer-Measured Physical Activity. Triaxial accelerometers (Actigraph ${ }^{\circledR}$, model wGT3X-BT; ActiGraph LLC, Pensacola, FL) objectively captured physical activity frequency, duration, and intensity (sampling rate of $100 \mathrm{~Hz}$ and 1-second epoch). Accelerometer recorded counts per minute were classified into physical activity intensity levels using Freedson adult cut-points (i.e., Light: 100-1951, Moderate: 1952-5724, Vigorous: >5724) (Freedson, Melanson, \& Sirard, 1998). Using the respective cut-points we also created two summed variables, moderate-to-vigorous intensity physical activity and light-to-vigorous intensity physical activity (total physical activity). Light-to-vigorous intensity physical activity was included as previous research has found that including light physical activity may better reflect the true associations and agreement between self-report and objectively measured physical activity (Cerin et al., 2012, 2016).

Accelerometer non-wear time was defined by $90 \mathrm{~min}$ of consecutive zero counts with a two-minute tolerance (Choi, Liu, Matthews, \& Buchowski, 2011). Valid wear time was determined if a participant had 10-hours or more per day (Holliday et al., 2017) for at least three days. With the exception of vigorous-intensity physical activity, the day-to-day intraclass correlation for accelerometer counts estimated for at least three valid consecutive days was .80 (Supplementary Material [available online]). ActiGraph ${ }^{\circledR}$ wGT3X-BT is a tri-axial accelerometer that has been found to be reliable in free-living situations (Aadland \& Ylvisåker, 2015) and has been validated in adults using indirect calorimetry (SantosLozano et al., 2013) and doubly labelled water (Chomistek et al., 2017) as criterion measures.

GPS Monitoring. GPS monitors (model: Qstarz BT-Q1000XT ${ }^{\circledR}$; Qstarz International Inc., Taiwan) captured the geographical location of participants' outdoor activity. The Qstarz BT-Q1000XT ${ }^{\circledR}$ has been used to monitor location-based physical activity in previous research (Jansen et al., 2018). The Qstarz GPS monitor has a median dynamic positional error of $2.9 \mathrm{~m}$ (Schipperijn et al., 2014). The GPS monitor was set to record latitude, longitude, height above sea level, speed $(\mathrm{km} / \mathrm{hr})$, and the coordinate log settings were set to 5-second epochs. We chose 5-second epochs due to shorter times resulting in the internal data storage reaching capacity before 7-days of monitoring.

Accelerometer/GPS-Measured Neighborhood Physical Activity. Accelerometer/GPS data were linked using the Personal Activity Location Measurement System (PALMS; ucsd-palmsproject.wikispaces.com). Missing GPS data were replaced by the last known valid point for up to $10 \mathrm{~min}$, and invalid GPS points were identified as points with a speed of more than $130 \mathrm{~km} / \mathrm{hr}$, a change in elevation of more than $1000-\mathrm{m}$, or if the minimum change in distances between three fixes was $10 \mathrm{~m}$. Invalid points were removed. As location was the main variable of interest, when merging the data, we merged the accelerometer data to the GPS data. PALMS merged the valid GPS data to the accelerometer data using synchronized time stamps. These merged data were uploaded to a custom-built PostgreSQL database and combined with Calgary neighborhood administrative boundary and the 400- and 800-m radial buffers to create the relevant accelerometer/GPS physical activity intensity variables. Administrative boundary shapefiles were publicly available from the city of Calgary. Thus, the GPS and accelerometer data together estimated light-, moderate-, vigorous-, moderate-to-vigorous intensity physical activity, and total physical activity undertaken within the neighborhood administrative boundary, and within 400-m and 800-m of the participant's home. The merged accelerometer/GPS physical activity variables were summed and divided by the number of valid days to obtain an estimate of daily accelerometer/GPS physical activity.

\section{Statistical Analysis}

Descriptive statistics including means (and Standard Deviations $[S D])$ and frequencies were estimated for all sample characteristics.

Concurrent Validity Assessment. Using Bland-Altman plots, we estimated the mean (95\% Confidence Interval [CI]) differences, and $95 \%$ limits of agreement, between the N-IPAQ and accelerometer/GPS measured daily minutes of neighborhood total and moderate-to-vigorous physical activity. Positive mean difference values represented an overestimate and negative mean difference values represented an underestimate of N-IPAQ reported values relative to estimated accelerometer/GPS measured physical activity. To further inform our assessment of the N-IPAQ's concurrent validity, using Spearman rank correlations $(r)$ we estimated the linear associations between the N-IPAQ and accelerometer/GPS measured neighborhood physical activity. Bland-Altman plots and Spearman rank correlations were estimated for each of three neighborhood sizes (i.e., administrative boundary, 400-m radial buffer, and 800-m radial buffer). Prior to the Bland-Altman plots, normality in the distribution of the mean differences between total accelerometer and N-IPAQ physical activity was assessed using 
Shapiro-Wilk tests. All statistical analyses were undertaken using STATA $^{\circledR}$ version 14.2 (StataCorp, TX, USA).

\section{Results}

\section{Sample Characteristics}

For three of the 75 participants, no valid wear times were captured on any of the 7 days of wear time (i.e., $<10$ hours). Another nine participants were excluded because there were insufficient numbers of valid days (i.e., $<3$ days). The mean (range) of valid days was 5.3 (minimum $=1$ and maximum $=7$ ). Using accelerometer merged to GPS data only 24 participants had 7 valid wear days. Therefore, the data from participants with less than 7 days of valid wear days provided estimates of last 7 days physical activity. Moreover, the Shapiro-Wilk tests suggested that the differences between total accelerometer and self-reported physical activity had a non-normal distribution and upon further investigation, two participants selfreported more than 5 hours a day of neighborhood physical activity relative to their accelerometer-measured physical activity. The calculated daily neighborhood-based physical activity of the two participants was 7 and 9 hours, taking into account hours of daily living (e.g., sleep, work, eating, and grooming) these values were deemed erroneous and therefore were subsequently excluded from the analysis. Moreover, including these outliers in the analysis resulted in marginal differences in estimates of agreement. The mean age of the remaining sample $(n=61)$ was 56 years $(S D=14)$ and two-thirds of the participants were female (67\%). A high percentage $(84 \%)$ of the sample had a university degree, all participants had access to a motor vehicle for personal use and most of the participants had access to a bicycle for their own use (84\%) (Table 1).

\section{Correspondence Between N-IPAQ and Accelerometer/GPS Physical Activity by Neighbourhood Boundary Definitions}

No Neighborhood Boundary. Total daily physical activity minutes captured by the N-IPAQ were underestimated relative to total daily minutes of accelerometer-measured physical activity with a mean difference (N-IPAQ minus accelerometer) of $-81.0 \mathrm{~min}$ (95\% CI: -109.5 to -52.6 ), and $95 \%$ limits of agreement of -298.9 to 136.9 min (Figure 1a). Total daily physical activity minutes captured by the N-IPAQ were overestimated relative to daily minutes of accelerometer-measured moderate-to-vigorous physical activity with a clear downward trend as average physical activity increased and a mean difference of $68.3 \mathrm{~min}$ (95\% CI: 43.1 to 93.5), and $95 \%$ limits of agreement of -124.5 to $261.1 \mathrm{~min}$ (Figure 2a). N-IPAQ captured neighborhood vigorous-intensity physical activity and overall accelerometer-measured vigorous-intensity physical activity was positively correlated $(r=.33 ; 95 \%$ CI: .09 to .54$)$ (Table 2).

Administrative Neighborhood Boundary. Total daily physical activity minutes captured by the N-IPAQ demonstrated agreement with total daily minutes of administrative neighborhood-based accelerometer/GPS measured physical activity (mean difference: $-14.7 \mathrm{~min}$; $95 \%$ CI: -41.0 to 11.5 , and; $95 \%$ limits of agreement of -215.8 to $18.3 \mathrm{~min}$ ) (Figure 1b). Daily moderate-to-vigorous physical activity minutes captured by the N-IPAQ was overestimated compared to administrative neighborhood-based accelerometer/ GPS measured moderate-to-vigorous physical activity (mean
Table 1 Sample Demographic and Physical Activity Characteristics for Participants $(n=61)$ Completing a Self-Administered N-IPAQ

\begin{tabular}{lc}
\hline Characteristic & Total \\
\hline Age in years, $M(S D)$ & $55.7(14.1)$ \\
Female, $n(\%)$ & $41(67.2)$ \\
Dependents living in the home, $n(\%)$ & \\
$\quad$ One or more <6 yrs old & $11(18.0)$ \\
$\quad$ One or more 6-18 yrs old & $10(16.4)$ \\
Dogs living in the home, $n(\%)$ & $22(36.1)$ \\
Had access to a motor vehicle for & $61(100.0)$ \\
personal use, $n(\%)$ & \\
Had access to a bicycle for & $51(83.6)$ \\
personal use, $n(\%)$ & \\
Highest level of education, $n(\%)$ & \\
$\quad$ Less than University & $10(16.4)$ \\
University & $51(83.6)$ \\
N-IPAQ estimate, $M$ min/day $(S D)$ & \\
Total walking & $58.5(51.8)$ \\
Moderate physical activity & $29.0(59.5)$ \\
Moderate physical activity + total walking & $87.5(84.9)$ \\
Vigorous physical activity & $31.4(48.3)$ \\
MVPA & $60.4(78.2)$ \\
MVPA + total walking & $118.9(94.2)$ \\
Total physical activity & $122.2(95.1)$
\end{tabular}

Accelerometer estimated physical activity,

$M \min /$ day $(S D)$

No restriction

Moderate

$50.6(25.4)$

Vigorous

$3.3(4.7)$

MVPA

$53.9(27.5)$

Total

$203.2(59.1)$

Within administrative boundary

Moderate

$29.5(16.7)$

Vigorous

$1.5(2.1)$

MVPA

$30.9(17.5)$

Total

$136.9(57.5)$

Within 800-m radial buffer

Moderate

$28.2(16.6)$

Vigorous

$1.5(2.2)$

MVPA

$29.7(17.3)$

Total

$132.7(59.7)$

Within 400-m radial buffer

Moderate

$24.3(14.2)$

Vigorous

$1.2(1.8)$

MVPA

$25.5(14.9)$

Total

$124.0(61.2)$

Note. $\mathrm{N}-\mathrm{IPAQ}=$ Neighbourhood International Physical Activity Questionnaire; $M=$ mean; $S D=$ Standard deviation; $\mathrm{MVPA}=$ moderate-to-vigorous intensity physical activity.

difference: $91.2 \mathrm{~min}$; 95\% CI: 67.2 to 115.3 , and; $95 \%$ limits of agreement of -92.8 to $275.3 \mathrm{~min}$ ), this overestimation increases as average physical activity increased (Figure 2b). 
A

$2 / 61=3.28 \%$ outside the limits of agreement

$95 \%$ limits of agreement (-298.9 to 136.9)

Mean difference $-81.0(95 \% \mathrm{Cl}:-109.5$ to -52.6$)$

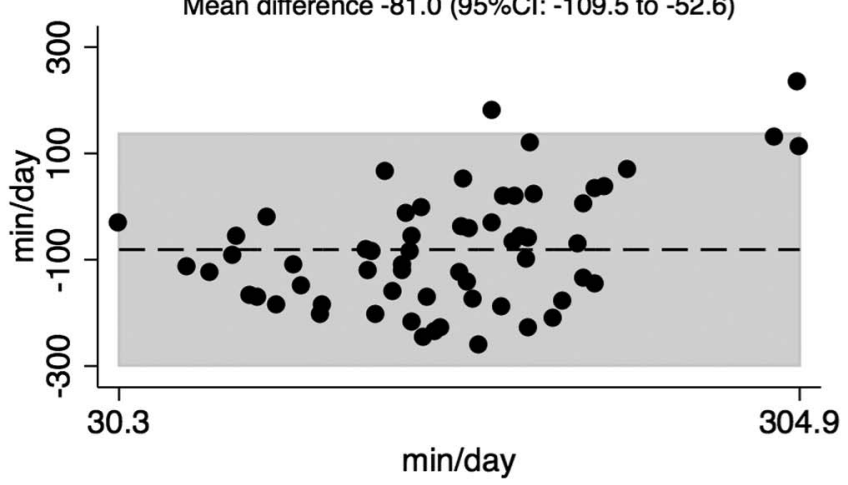

C

$3 / 61=4.92 \%$ outside the limits of agreement $95 \%$ limits of agreement (-216.3 to 212.6 ) Mean difference -1.9 (95\% Cl: -29.9 to 26.2$)$

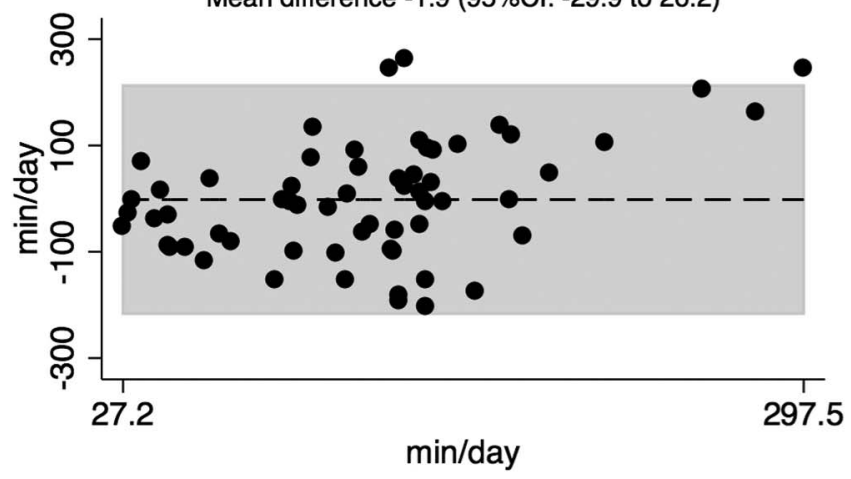

$B$

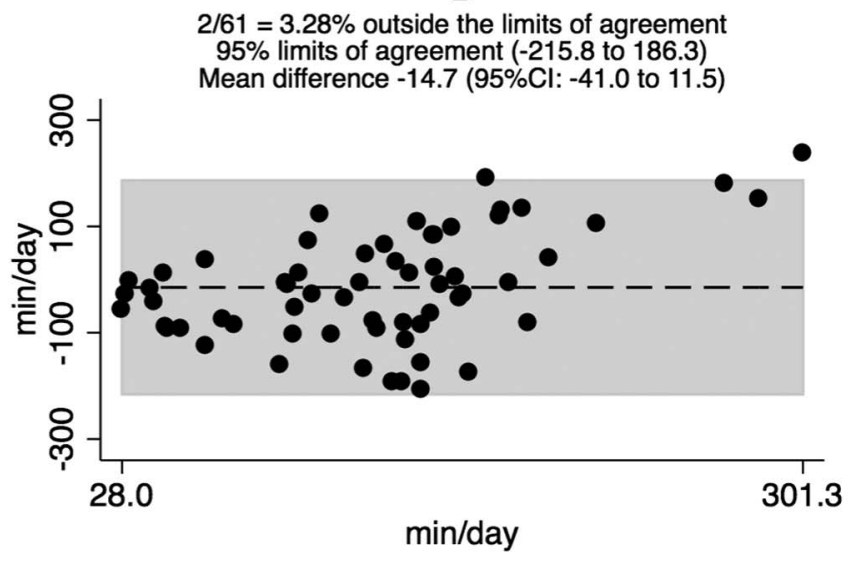

D

$1 / 61=1.64 \%$ outside the limits of agreement $95 \%$ limits of agreement (-213.8 to 192.7) Mean difference $-10.6(95 \% \mathrm{Cl}:-37.1$ to 16.0

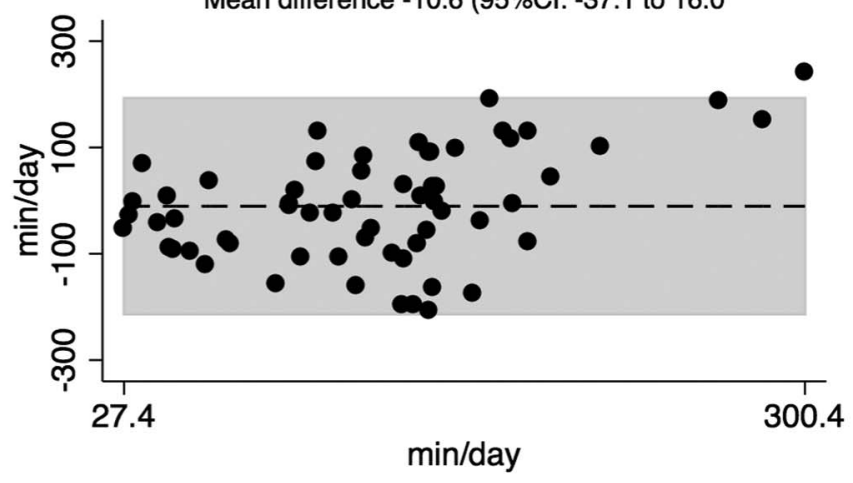

Figure 1 - Bland-Altman plot assessing agreement between total accelerometer based physical activity (min/day) and differing neighborhood definitions $(n=61)$. Data is displayed as N-IPAQ minus accelerometer. a) No restriction, b) Administrative boundary, c) 400-m radial buffer, d) 800-m radial buffer. Shaded area indicates limits of agreement, dashed line indicates mean difference.

N-IPAQ assessed total walking, and moderate physical activity plus walking were positively correlated with administrative neighborhood-based accelerometer/GPS-measured moderateintensity physical activity $(r=.37,95 \% \mathrm{CI}: .13$ to .59 ; and $r=.30 ; 95 \%$ CI: .05 to .51 , respectively) (Table 2). Moreover, N-IPAQ assessed vigorous-intensity physical activity was positively correlated with administrative neighborhood-based accelerometer/ GPS-measured vigorous-intensity physical activity $(r=.43 ; 95 \%$ CI: .20 to .62).

Neighbourhood Within 400-m Radius of Home. We found agreement between N-IPAQ assessed total daily physical activity minutes and total daily minutes of accelerometer/GPS measured physical activity estimated within 400-m of home (mean difference: $-1.9 \mathrm{~min}$; $95 \% \mathrm{CI}$ : -29.9 to 26.2 , and; $95 \%$ limits of agreement of -216.3 to $212.6 \mathrm{~min}$ ) (Figure 1c). However, for N-IPAQ moderate-to-vigorous intensity physical activity relative to accelerometer/GPS moderate-to-vigorous physical activity within 400-m of home there is a clear downward trend in the Bland and Altman plot indicating increasing overestimation (mean difference: $96.7 \mathrm{~min}$; $95 \% \mathrm{CI}$ : 72.2 to 121.2 , and; $95 \%$ limits of agreement of -90.6 to $284.0 \mathrm{~min}$ ) (Figure 2c). N-IPAQ captured vigorous-intensity physical activity was positively correlated with accelerometer/GPS vigorous-intensity physical activity estimated within 400-m of home ( $r=.32$; 95\% CI: .08 to .53) (Table 2).
Neighbourhood Within 800-m Radius of Home. The mean difference between N-IPAQ and accelerometer/GPS total daily physical activity within 800-m of home showed agreement (mean difference: $-10.6 \mathrm{~min}$; $95 \% \mathrm{CI}$ : -37.1 to 16.0 , and; $95 \%$ limits of agreement of -213.8 to $192.7 \mathrm{~min}$ ) (Figure 1d). Compared with accelerometer/GPS, the N-IPAQ overestimated total daily minutes of physical activity within 800-m of home (mean difference: 92.5 min; $95 \%$ CI: 68.3 to 116.7 , and; $95 \%$ limits of agreement of -92.5 to $116.7 \mathrm{~min}$ ) with a downward trend line shown (Figure $2 \mathrm{~d}$ ). For the 800-m neighborhood buffer, N-IPAQ assessed total walking was positively correlated with accelerometer/GPS moderateintensity physical activity estimated within 800 -m of home $(r=.29$; 95\% CI: .04 to .51), and N-IPAQ vigorous-intensity physical activity was positively correlated with accelerometer/GPS vigorousintensity physical activity estimated within $800 \mathrm{~m}$ of home $(r=.41$; 95\% CI: .18 to .60) (Table 2).

\section{Discussion}

We have previously demonstrated that the N-IPAQ has acceptable test-retest reliability and convergent validity (i.e., significant associations with neighborhood walkability) (Frehlich et al., 2018). Contributing to this previous evidence, the present analysis demonstrates that for the most part, the N-IPAQ has concurrent validity 
A

$3 / 61=4.92 \%$ outside the limits of agreement $95 \%$ limits of agreement (-124.5 to 261.1$)$ Mean difference 68.3 (95\% $\mathrm{Cl}: 43.1$ to 93.5$)$

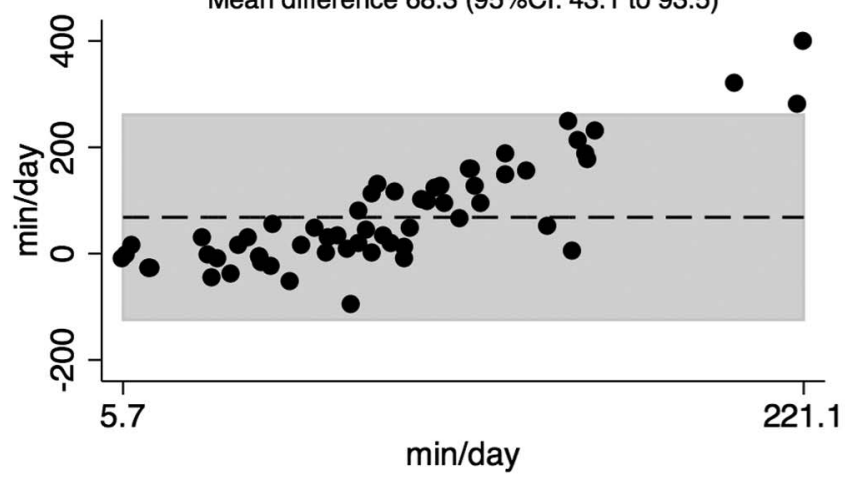

C

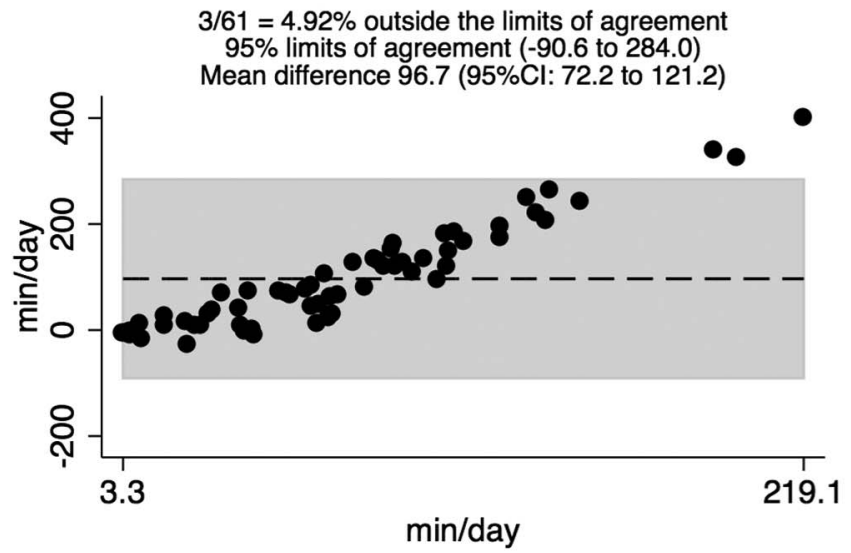

B

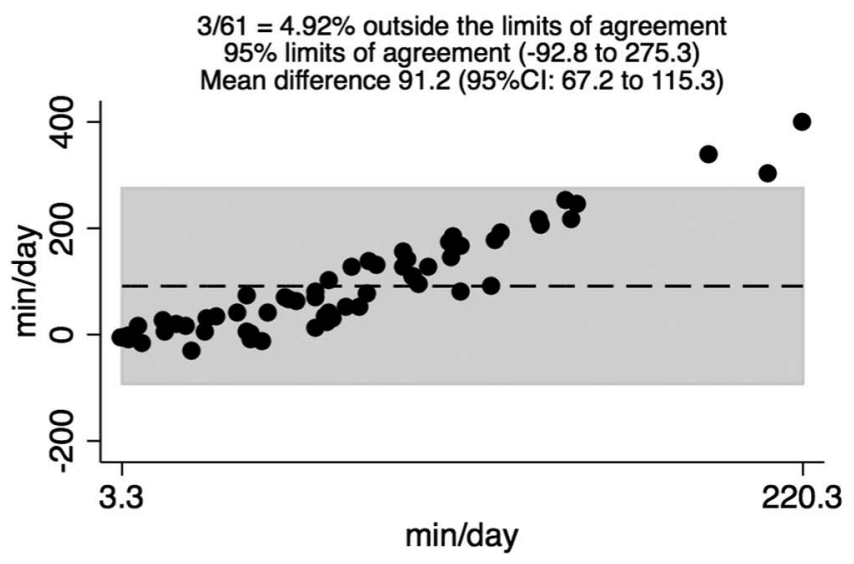

D

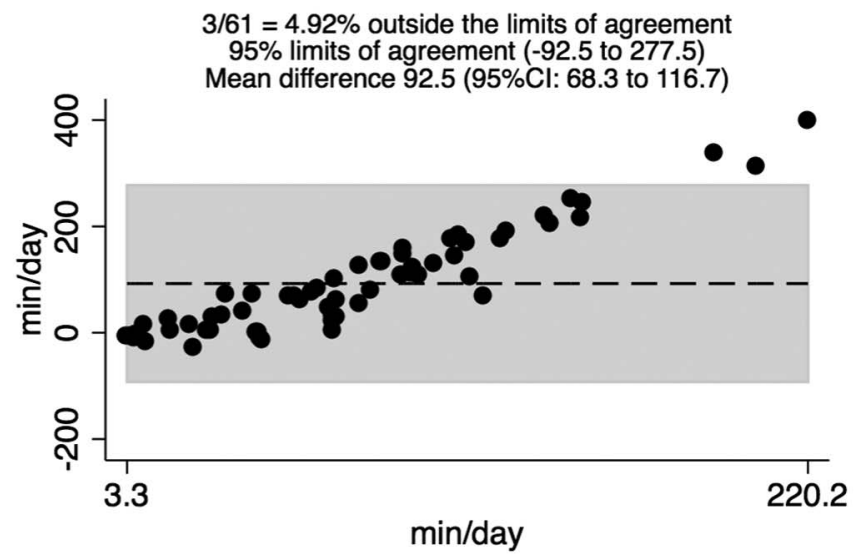

Figure 2 - Bland-Altman plot assessing agreement between moderate-to-vigorous intensity accelerometer based physical activity (min/day) and differing neighborhood definitions $(n=61)$. Data is displayed as N-IPAQ minus accelerometer. a) No restriction, b) Administrative boundary, c) 400-m radial buffer, d) 800-m radial buffer. Shaded area indicates limits of agreement, dashed line indicates mean difference.

when compared with accelerometer/GPS measured physical activity. Notably, our findings also suggest that a neighborhood definition of a 400-m radius from home demonstrated better agreement than neighborhood physical activity undertaken within an 800-m radius of home or for the administrative boundary.

The agreement for physical activity estimated for the N-IPAQ was sensitive to the size of the neighborhood boundary used to estimate accelerometer/GPS total daily physical activity. When no neighborhood boundary was defined, the accelerometer captured, on average, 81 more daily minutes of physical activity when compared to the N-IPAQ. However, this difference was reduced to just 15-m/day when accelerometer data were spatially restricted to the administrative boundary, notably this difference was attenuated even further when accelerometer data were spatially restricted to the 800-m and 400-m radial buffers (i.e., total daily physical activity produced a mean difference of 11 and $2 \mathrm{~min}$, respectively). The finding that the agreement between the N-IPAQ captured and accelerometer-measured physical activity became stronger as the spatially restricted neighborhood area became smaller, noteworthy, given that the N-IPAQ did not explicitly define the neighborhood for the respondent. Participants might more accurately perceive their neighborhood physical activity at distances closer to their homes, since the home is likely the starting and end point (i.e., an "anchor") for many neighborhood-based physical activity episodes
(Millward, Spinney, \& Scott, 2013). However, perceived distance to neighborhood destinations that are close to a participant's home may be overestimated whereas the distance to farther neighborhood destinations may be underestimated (McCormack, Cerin, Leslie, Du Toit, \& Owen, 2008). Moreover, participants may perceive their overall neighborhood as a smaller more localized area immediately surrounding their home (i.e., within $400 \mathrm{~m}$ ) (Smith, Gidlow, Davey, \& Foster, 2010). A study of adults living in England found that perceived neighborhood areas were approximately $40 \%$ ( 400-m) of objectively estimated $1 \mathrm{~km}$ radial or network buffers (Smith et al., 2010). Moreover, a systematic review of built environment correlates and walking in older adults found that the most frequently reported perceived neighborhood definition was a 10-20-m walk from home (Barnett et al., 2017) which, based on an average walking speed of older adults, equates to about a 550 to $1100-\mathrm{m}$ from home (Studenski, Perera, Patel, \& Al, 2011). The N-IPAQ provides an accurate estimate of self-reported neighborhood physical activity undertaken close to home however, future studies may wish to explore the impact of using predefined neighborhood definitions (e.g., 100-m, 1000-m) on the accuracy of respondent self-reporting of their neighborhood physical activity.

Similar to previous studies (Cerin et al., 2012, 2016), the addition of accelerometer-measured light-intensity physical activity (defined as count/minute of 100-1951) as part of the total 
Table 2 Spearman Rank Correlation Coefficients Between Average Minutes a Day of Accelerometer-Based and Self-Reported Neighbourhood Physical Activity, Using Different Neighbourhood Definitions $(n=61)$

\begin{tabular}{|c|c|c|c|c|}
\hline \multirow[b]{2}{*}{ N-IPAQ Estimate } & \multicolumn{4}{|c|}{ Accelerometer-Estimated Physical Activity } \\
\hline & Moderate & Vigorous & MVPA & Total \\
\hline \multicolumn{5}{|l|}{ No restriction } \\
\hline Total walking & $.21(-.04$ to .44$)$ & & $.18(-.08$ to .41$)$ & \\
\hline Moderate physical activity & $-.10(-.3$ to .16$)$ & & $-.08(-.33$ to .17$)$ & \\
\hline Moderate physical activity + total walking & $.11(-.15$ to .35$)$ & & $.09(-.17$ to .33$)$ & \\
\hline Vigorous physical activity & & $.33(.09$ to .54$)$ & $.14(-.12$ to .38$)$ & \\
\hline MVPA & & & $.05(-.20$ to .30$)$ & \\
\hline MVPA + total walking & & & $.11(-.15$ to .35$)$ & \\
\hline Total physical activity & & & $.10(-.15$ to .35$)$ & $.04(-.21$ to .29$)$ \\
\hline \multicolumn{5}{|l|}{ Within administrative boundary } \\
\hline Total walking & $.37(.13$ to .59$)$ & & $.32(.08$ to .53$)$ & \\
\hline Moderate physical activity & $.02(-.24$ to .27$)$ & & $.01(-.24$ to .26$)$ & \\
\hline Moderate physical activity + total walking & $.30(.05$ to .51$)$ & & $.26(.01$ to .48$)$ & \\
\hline Vigorous physical activity & & $.43(.20$ to .62$)$ & $.20(-.06$ to .43$)$ & \\
\hline MVPA & & & $.18(-.08$ to .42$)$ & \\
\hline MVPA + total walking & & & $.30(.05$ to .51$)$ & \\
\hline Total physical activity & & & $.27(.03$ to .49$)$ & $.17(-.08$ to .41$)$ \\
\hline \multicolumn{5}{|l|}{ Within $800-\mathrm{m}$ radial buffer } \\
\hline Total walking & $.29(.04$ to .51$)$ & & $.26(.00$ to .48$)$ & \\
\hline Moderate physical activity & $-.01(-.26$ to .24$)$ & & $-.02(-.27$ to .23$)$ & \\
\hline Moderate physical activity + total walking & $.24(-.01$ to .46$)$ & & $.21(-.05$ to .44$)$ & \\
\hline Vigorous physical activity & & $.41(.18$ to .60$)$ & $.19(-.07$ to .42$)$ & \\
\hline MVPA & & & $.15(-.10$ to .39$)$ & \\
\hline MVPA + total walking & & & $.25(.00$ to .47$)$ & \\
\hline Total physical activity & & & $.23(-.02$ to .46$)$ & $.18(-.08$ to .41$)$ \\
\hline \multicolumn{5}{|l|}{ Within $400-\mathrm{m}$ radial buffer } \\
\hline Total walking & $.19(-.06$ to .43$)$ & & $.16(-.10$ to .40$)$ & \\
\hline Moderate physical activity & $.02(-.24$ to .27$)$ & & $.02(-.23$ to .27$)$ & \\
\hline Moderate physical activity + total walking & $.17(-.09$ to .40$)$ & & $.14(-.12$ to .38$)$ & \\
\hline Vigorous physical activity & & $.32(.08$ to .53$)$ & $.17(-.08$ to .41$)$ & \\
\hline MVPA & & & $.12(-.13$ to .36$)$ & \\
\hline MVPA + total walking & & & $.16(-.09$ to .40$)$ & \\
\hline Total physical activity & & & $.15(-.11$ to .39$)$ & $.11(-.14$ to .35$)$ \\
\hline
\end{tabular}

Note. Data presented as $r(95 \%$ CI). N-IPAQ = Neighbourhood International Physical Activity Questionnaire; MVPA = Moderate-to-vigorous intensity physical activity.

accelerometer-measured physical activity outcome, greatly improved the measures of agreement between self-reported and accelerometermeasured physical activity. Moreover, without including light-intensity physical activity, agreement clearly demonstrates a strong upward trend which violates the assumptions of the Bland and Altman assessment. This finding is important to note since the IPAQ was originally designed to capture only moderate-to-vigorous physical activity and subsequent validity research using accelerometers was based on matching these intensities (Craig et al., 2003). Moreover, the IPAQ may overestimate physical activity intensity; therefore, steps have been taken to control for (Cerin et al., 2012), or add in (Cerin et al., 2016) light-intensity physical activity to improve the accuracy of comparisons between self-reported and objectively measured physical activity. Moreover, when we restricted the accelerometer data to the neighborhood administrative boundary, six, albeit low to moderate correlations with N-IPAQ outcomes
( $r=.26 ; 95 \%$ CI: .00 to .48 to $.43 ; 95 \%$ CI: .20 to .62 ) were found; more than other neighborhood boundary definitions. Although not specific to any neighborhood definition, others have found weaker positive correlations between IPAQ moderate-physical activity plus walking and accelerometer-measured moderate-intensity physical activity among adults (Cerin et al., 2016). Our findings might suggest that restricting self-reported physical activity to a geographical area, such as the neighborhood, results in stronger correlations with accelerometer-measured physical activity. However, the $\mathrm{N}$ IPAQ should be used with caution when assessing individual data (compared with aggregate neighborhood data) as our findings suggest that on average participants overestimate their total neighborhood physical activity using the N-IPAQ in comparison to accelerometer-measured physical activity.

The main strength of our study was the inclusion of objectively measured location-specific physical activity with location-specific 
self-reported physical activity both from the last 7 days to assess concurrent validity. This component of our study is novel given the lack of evidence on the measurement validity of self-report neighborhood based physical activity questionnaires. There were, however, methodologic limitations that should be considered in the interpretation of our findings. Our sample was based on convenience (i.e., previous study participants) and purposive sampling (i.e., moderate SES neighborhoods in Calgary only) limiting the generalizability of our findings. Moreover, the small sample size precluded stratified analyses by participant sociodemographic characteristics. Additionally, although participants were instructed to not deviate from their normal physical activities or routine during the study, it is possible that some participants increased their physical activity due to being monitored and others might have been more cognizant of the physical activity they were undertaking during the week of data collection. While this limits generalizability, this should not impact the agreement between the two measures. If participants, were more cognizant of their physical activity during the week of monitoring, they may more accurately recall their physical activity on the N-IPAQ leading to higher agreement with the accelerometer data. Furthermore, recent research has identified limitations in the use of neighborhood buffers (like those used in this study), and there are calls to focus on "activity spaces" (i.e., individually-defined areas based on GPS data) which might provide a more accurate geographical representation of movement and activity patterns (Hirsch, Winters, Ashe, Clarke, \& McKay, 2016; Howell, Farber, Widener, \& Booth, 2017). While outside the scope of the current study, future research should consider using activity spaces when comparing accelerometer and self-reported neighborhood-based physical activity outcomes. Therefore, for the estimation of activity spaces, travel/ movement speed and distances, intensity and volume, specific locations of activity, accelerometer/GPS data provides important information. However, the N-IPAQ would provide more relevant data where there is interest in specific context or location (as perceived by the participant), such as the "neighborhood", and/or there is interest in specific types or purpose of physical activity (e.g., walking for transportation vs. recreation, cycling for transportation vs. recreation, running/jogging, etc.).

Preliminary evidence presented here and elsewhere (Frehlich et al., 2018), suggest that the N-IPAQ is a reliable and valid tool for estimating neighborhood-based physical activity in adults; however, further evaluation of the questionnaire's measurement properties is needed. The N-IPAQ is an adaptation of the IPAQ, designed specifically to measure neighborhood-based physical activity only. Thus, we acknowledge that our adaptation builds upon the many decades of research and the expert advice that informed the development of the original IPAQ (Craig et al., 2003). The N-IPAQ may provide a convenient and useful tool for capturing location-based physical activity in investigations of neighborhood-built environment correlates of physical activity in adult populations.

\section{Acknowledgments}

The contributions of the co-investigators and collaborators on the Pathways to Health Study is greatly appreciated. This study was part of the Pathways to Health project funded by the Canadian Institutes of Health Research (CIHR; MOP-126133). Additional funding support was provided by an O'Brien Centre for Public Health Catalyst Grant, a University Research Grant Committee Seed Grant, and a CIHR Foundations Scheme Grant (FDN-154331).

\section{References}

Aadland, E., \& Ylvisåker, E. (2015). Reliability of the Actigraph GT3X+ accelerometer in adults under free-living conditions. PLoS One, 10(8), e0134606. PubMed ID: 26274586 doi:10.1371/journal.pone. 0134606

Bae, W.K., Cho, B., \& Son, K.Y. (2015). Validity and reliability of the Korean version of neighborhood physical activity questionnaire. Korean Journal of Family Medicine, 36(3), 135-140. PubMed ID: 26019763 doi:10.4082/kjfm.2015.36.3.135

Barnett, D.W., Barnett, A., Nathan, A., Van Cauwenberg, J., Cerin, E., Van Cauwenberg, J., \& Cerin, E. (2017). Built environmental correlates of older adults' total physical activity and walking: A systematic review and meta-analysis. The International Journal of Behavioral Nutrition and Physical Activity, 14(1), 103. PubMed ID: 28784183 doi:10.1186/s12966-017-0558-z

Calise, T.V., Heeren, T., DeJong, W., Dumith, S.C., \& Kohl, H.W. (2013). Do neighborhoods make people active, or do people make active neighborhoods? Evidence from a planned community in Austin, Texas. Preventing Chronic Disease, 10(2), E102. doi:10.5888/ pcd10.120119

Cerin, E., Barnett, A., Cheung, M., Sit C, H.P., Macfarlane, D.J., \& Chan, W.M. (2012). Reliability and validity of the IPAQ-L in a sample of Hong Kong urban older adults: Does neighborhood of residence matter? Journal of Aging and Physical Activity, 20(4), 402-420. PubMed ID: 22186607 doi:10.1123/japa.20.4.402

Cerin, E., Cain, K.L., Oyeyemi, A.L., Owen, N., Conway, T.L., Cochrane, T., . . . Sallis, J.F. (2016). Correlates of agreement between accelerometry and self-reported physical activity. Medicine and Science in Sports and Exercise, 48, 1075-1084. doi:10.1249/MSS .0000000000000870

Cerin, E., Nathan, A., van Cauwenberg, J., Barnett, D.W., Barnett, A., \& Council on Environment and Physical Activity (CEPA)-Older Adults Working Group. (2017). The neighbourhood physical environment and active travel in older adults: A systematic review and metaanalysis. The International Journal of Behavioral Nutrition and Physical Activity, 14(1), 15. PubMed ID: 28166790 doi:10.1186/ s12966-017-0471-5

Cerin, E., Saelens, B.E., Sallis, J.F., \& Frank, L.D. (2006). Neighborhood environment walkability scale: Validity and development of a short form. Medicine and Science in Sports and Exercise, 38(9), 1682-1691. PubMed ID: 16960531 doi:10.1249/01.mss.0000227639.83607.4d

Choi, L., Liu, Z., Matthews, C.E., \& Buchowski, M.S. (2011). Validation of accelerometer wear and nonwear time classification algorithm. Medicine and Science in Sports and Exercise, 43(2), 357-364. PubMed ID: 20581716 doi:10.1249/MSS.0b013e3181ed61a3

Chomistek, A.K., Yuan, C., Matthews, C.E., Troiano, R.P., Bowles, H.R., Rood, J., . . Bassett, D.R. (2017). Physical activity assessment with the ActiGraph GT3X and doubly labeled water. Medicine and Science in Sports and Exercise, 49(9), 1935-1944. PubMed ID: 28419028 doi:10.1249/MSS.0000000000001299

Christian, H.E., Bull, F.C., Middleton, N.J., Knuiman, M.W., Divitini, M.L., Hooper, P., . . G Giles-Corti, B. (2011). How important is the land use mix measure in understanding walking behaviour? Results from the RESIDE study. The International Journal of Behavioral Nutrition and Physical Activity, 8(1), 55. doi:10.1186/1479-58688-55

Craig, C.L., Marshall, A.L., Sjöström, M., Bauman, A.E., Booth, M.L., Ainsworth, B.E., . . O Oja, P. (2003). International physical activity questionnaire: 12-Country reliability and validity. Medicine and Science in Sports and Exercise, 35(8), 1381-1395. PubMed ID: 12900694 doi:10.1249/01.MSS.0000078924.61453.FB 
Doma, K., Speyer, R., Leicht, A.S., \& Cordier, R. (2017). Comparison of psychometric properties between usual-week and past-week selfreported physical activity questionnaires: A systematic review. The International Journal of Behavioral Nutrition and Physical Activity, 14(1), 10. PubMed ID: 28137268 doi:10.1186/s12966-017-0470-6

Ferdinand, A.O., Sen, B., Rahurkar, S., Engler, S., \& Menachemi, N. (2012). The relationship between built environments and physical activity: A systematic review. American Journal of Public Health, 102(10), e7-e13. doi:10.2105/AJPH.2012.300740

Freedson, P.S., Melanson, E., \& Sirard, J. (1998). Calibration of the computer science and applications, Inc. accelerometer. Medicine and Science in Sports and Exercise, 30(5), 777-781. PubMed ID: 9588623 doi:10.1097/00005768-199805000-00021

Frehlich, L., Friedenreich, C., Nettel-Aguirre, A., \& McCormack, G.R. (2018). Test-retest reliability of a modified International Physical Activity Questionnaire (IPAQ) to capture neighbourhood physical activity. Journal of Human Sport and Exercise, 13(1), 174-187. doi:10.14198/jhse.2018.131.17

Giles-Corti, B., Bull, F., Knuiman, M., McCormack, G.R., Van Niel, K., Timperio, A., .. . Boruff, B. (2013). The influence of urban design on neighbourhood walking following residential relocation: Longitudinal results from the RESIDE study. Social Science \& Medicine (1982), 77(1), 20-30. doi:10.1016/j.socscimed.2012.10.016

Giles-Corti, B., Timperio, A., Cutt, H., Pikora, T.J., Bull, F.C., Knuiman, M., ... Shilton, T. (2006). Development of a reliable measure of walking within and outside the local neighborhood: RESIDE's Neighborhood physical activity questionnaire. Preventive Medicine, 42(6), 455-459. PubMed ID: 16574208 doi:10.1016/j.ypmed.2006.01.019

Hirsch, J.A., Winters, M., Ashe, M.C., Clarke, P., \& McKay, H. (2016). Destinations that older adults experience within their GPS activity spaces relation to objectively measured physical activity. Environment and Behavior, 48(1), 55-77. PubMed ID: 26783370 doi:10. $1177 / 0013916515607312$

Holliday, K.M., Howard, A.G., Emch, M., Rodríguez, D.A., Rosamond, W.D., \& Evenson, K.R. (2017). Deriving a GPS monitoring time recommendation for physical activity studies of adults. Medicine and Science in Sports and Exercise, 49(5), 939-947. PubMed ID: 28009791 doi:10.1249/MSS.0000000000001190

Howell, N.A., Farber, S., Widener, M.J., \& Booth, G.L. (2017). Residential or activity space walkability: What drives transportation physical activity? Journal of Transport \& Health, 7, 160-171. doi:10.1016/j. jth.2017.08.011

Humpel, N., Owen, N., Leslie, E., Marshall, A.L., Bauman, A.E., \& Sallis, J.F. (2004). Associations of location and perceived environmental attributes with walking in neighborhoods. American Journal of Health Promotion: AJHP, 18(3), 239-242. PubMed ID: 30407068 doi:10.4278/0890-1171-18.3.239

Jansen, M., Kamphuis, C.B.M., Pierik, F.H., Ettema, D.F., \& Dijst, M.J. (2018). Neighborhood-based PA and its environmental correlates: A GIS- and GPS based cross-sectional study in the Netherlands. BMC Public Health, 18(1), 233. PubMed ID: 29426309 doi:10.1186/ s12889-018-5086-5

Kärmeniemi, M., Lankila, T., Ikäheimo, T., Koivumaa-Honkanen, H., \& Korpelainen, R. (2018). The built environment as a determinant of physical activity: A systematic review of longitudinal studies and natural experiments. Annals of Behavioral Medicine, 52(3), 239-251. doi:10.1093/abm/kax043

Krenn, P.J., Titze, S., Oja, P., Jones, A., \& Ogilvie, D. (2011). Use of global positioning systems to study physical activity and the environment: A systematic review. American Journal of Preventive Medicine, 41(5), 508-515. PubMed ID: 22011423 doi:10.1016/j.amepre.2011. 06.046
MacMillan, F., George, E.S., Feng, X., Merom, D., Bennie, A., Cook, A., . . A Astell-Burt, T. (2018). Do natural experiments of changes in neighborhood built environment impact physical activity and diet? A systematic review. International Journal of Environmental Research and Public Health, 15(2), 217. doi:10.3390/ijerph15020217

McCormack, G.R., Blackstaffe, A., Nettel-Aguirre, A., Csizmadi, I., Sandalack, B., Uribe, F., . . P Potestio, M. (2018). The Independent Associations between Walk Score ${ }^{\circledR}$ and Neighborhood Socioeconomic Status, Waist Circumference, Waist-To-Hip Ratio and Body Mass Index Among Urban Adults. International journal of environmental research and public health 15(6), 1226.

McCormack, G.R., Cerin, E., Leslie, E., Du Toit, L., \& Owen, N. (2008). Objective versus perceived walking distances to destinations: Correspondence and predictive validity. Environment and Behavior, 40(3), 401-425. doi:10.1177/0013916507300560

McCormack, G.R., Csizmadi, I., Friedenreich, C., McLaren, L., NettelAguirre, A., Potestio, M., \& Sandalack, B. (2013). Pathways to weight: The influence of neighbourhood environments on the weight status of adults. Canadian Institutes of Health Research, Open Operating Grant Competition.

McCormack, G.R., Friedenreich, C., Sandalack, B.A., Giles-Corti, B., Doyle-Baker, P.K., \& Shiell, A. (2012). The relationship between cluster-analysis derived walkability and local recreational and transportation walking among Canadian adults. Health \& Place, 18(5), 1079-1087. PubMed ID: 22652511 doi:10.1016/j.healthplace.2012. 04.014

McCormack, G.R., Shiell, A., Doyle-Baker, P., Friedenreich, C., Sandalack, B., \& Giles-Corti, B. (2009). Testing the reliability of neighborhood-specific measures of physical activity among Canadian adults. Journal of Physical Activity \& Health, 6(3), 367-373. PubMed ID: 19564667 doi:10.1123/jpah.6.3.367

McInerney, M., Csizmadi, I., Friedenreich, C.M., Uribe, F.A., NettelAguirre, A., McLaren, L., . . McCormack, G.R. (2016). Associations between the neighbourhood food environment, neighbourhood socioeconomic status, and diet quality: An observational study. $B M C$ Public Health, 16, 984. PubMed ID: 27633380 doi:10.1186/ s12889-016-3631-7

Millward, H., Spinney, J., \& Scott, D. (2013). Active-transport walking behavior: Destinations, durations, distances. Journal of Transport Geography, 28, 101-110. doi:10.1016/j.jtrangeo.2012.11.012

Sallis, J.F., Cerin, E., Conway, T.L., Adams, M.A., Frank, L.D., Pratt, M., ... Owen, N. (2016). Physical activity in relation to urban environments in 14 cities worldwide: A cross-sectional study. Lancet (London, England), 387(10034), 2207-2217. doi:10.1016/S01406736(15)01284-2

Santos-Lozano, A., Santín-Medeiros, F., Cardon, G., Torres-Luque, G., Bailón, R., Bergmeir, C., . . . Garatachea, N. (2013). Actigraph GT3X: Validation and determination of physical activity intensity cut points. International Journal of Sports Medicine, 34(11), 975-982. PubMed ID: 23700330 doi:10.1055/s-0033-1337945

Schipperijn, J., Kerr, J., Duncan, S., Madsen, T., Klinker, C.D., \& Troelsen, J. (2014). Dynamic accuracy of GPS receivers for use in health research: A novel method to assess GPS accuracy in real-world settings. Frontiers in Public Health, 2, 21. PubMed ID: 24653984 doi:10.3389/fpubh.2014.00021

Smith, G., Gidlow, C., Davey, R., \& Foster, C. (2010). What is my walking neighbourhood? A pilot study of English adults' definitions of their local walking neighbourhoods. The International Journal of Behavioral Nutrition and Physical Activity, 7(1), 34. doi:10.1186/1479-5868-7-34

Studenski, S., Perera, S., Patel, K., \& Al, E. (2011). Gait speed and survival in older adults. JAMA, 305(1), 50-58. PubMed ID: 21205966 doi:10. 1001/jama.2010.1923 
Sundquist, K., Eriksson, U., Kawakami, N., Skog, L., Ohlsson, H., \& Arvidsson, D. (2011). Neighborhood walkability, physical activity, and walking behavior: The Swedish Neighborhood and Physical Activity (SNAP) study. Social Science \& Medicine (1982), 72(8), 1266-1273. doi:10.1016/j.socscimed.2011.03.004

Troped, P.J., Wilson, J.S., Matthews, C.E., Cromley, E.K., \& Melly, S.J. (2010). The built environment and location-based physical activity.
American Journal of Preventive Medicine, 38(4), 429-438. PubMed ID: 20307812 doi:10.1016/j.amepre.2009.12.032

Wasfi, R., Steinmetz-Wood, M., \& Kestens, Y. (2017). Place matters: A longitudinal analysis measuring the association between neighbourhood walkability and walking by age group and population center size in Canada. PLoS One, 12(12), e0189472. PubMed ID: 29261706 doi:10.1371/journal.pone.0189472 